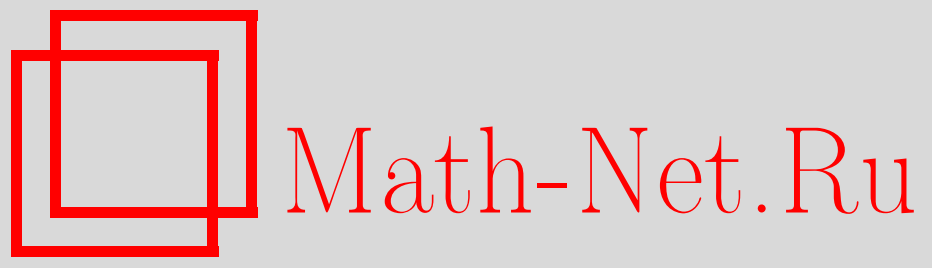

В. А. Зорич, Многомерная геометрия, функции очень многих переменных и вероятность, Теория вероятн. и ее примен., 2014, том 59, выпуск 3, 436-451

DOI: https://doi.org/10.4213/tvp4578

Использование Общероссийского математического портала Math-Net.Ru подразумевает, что вы прочитали и согласны с пользовательским соглашением http://www.mathnet.ru/rus/agreement

Параметры загрузки:

IP : 3.93 .64 .190

26 апреля 2023 г., 13:54:50

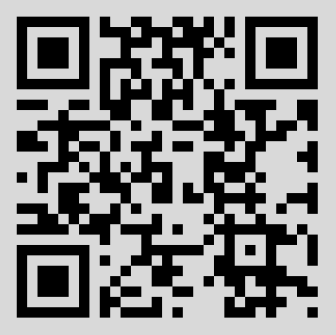




\section{МНОГОМЕРНАЯ ГЕОМЕТРИЯ, ФУНКЦИИ ОЧЕНЬ МНОГИХ ПЕРЕМЕННЫХ И ВЕРОЯТНОСТЬ ${ }^{1)}$}

Разнородные факты (из статистической физики, теории вероятностей, теории динамических систем, геометрии, анализа), фигурирующие в этой заметке, по сути своей специалистам известны, но независимо, по отдельности. Все, что мы делаем, состоит в их интерпретации и обсуждении как проявления одного и того же феномена концентрации меры, возникающего в многомерных пространствах и относящегося к функциям очень большого числа переменных. Такие функции имеют тенденцию быть почти постоянными с точки зрения наблюдателя.

Мы доказываем также одну общую теорему о концентрации объема многомерной области.

Ключевые слова и фразы: многомерная геометрия, концентрация меры, функции очень многих переменных, закон больших чисел, центральная предельная теорема, нормальное распределение.

\section{1. Объем многомерного тела, сфера, случайные векторы.}

Почти весь объем многомерного тела находится в малой окрестности границы тела. Это легко проверить на примерах куба и шара. Так, если с тысячемерного арбуза радиуса 1 метр снять корку толщины 1 см, то останется меньше тысячной доли исходного арбуза.

Если сферу $S^{n-1}(r) \subset \mathbf{R}^{n}$ ортогонально спроектировать на гиперплоскость, проходящую через центр сферы, то получится шар (дважды покрытый) той же размерности $n-1$ и того же радиуса $r$. Учитывая сказанное выше, можно заметить (пока на качественном уровне), что почти вся площадь сферы $S^{n-1}(r)$ при $n \gg 1$ сосредоточена в малой окрестности экватора - пересечения сферы и указанной гиперплоскости.

Сферу $S^{n-1}(r)$ радиуса $r$ с центром в начале координат $n$-мерного евклидова пространства $\mathbf{R}^{n}$ спроектируем теперь ортогонально на координатную ось. Получится отрезок $[-r, r]$. Зафиксируем отрезок $[a, b] \subset$ $[-r, r]$. Пусть $S[a, b]$ 一 площадь той части $S_{[a, b]}^{n-1}(r)$ сферы $S^{n-1}(r)$, которая проектируется в отрезок $[a, b]$. Подсчитав отношение $S[a, b] / S[-r, r]$,

* Московский государственный университет им. М. В. Ломоносова, Москва, Россия; e-mail: vzor@mccme.ru

1) Работа выполнена при поддержке РФФИ (гранты № 14-01-00709-а, 13-01-12417офи-м). 
т.е. вероятность $\operatorname{Pr}_{n}[a, b]$ того, что случайно выбранная точка сферы окажется в слое $S_{[a, b]}^{n-1}(r)$ над отрезком $[a, b]$ (в предположении, что точки распределены по сфере равномерно), найдем, что

$$
\operatorname{Pr}_{n}[a, b]=\frac{\int_{a}^{b}\left(1-(x / r)^{2}\right)^{(n-3) / 2} d x}{\int_{-r}^{r}\left(1-(x / r)^{2}\right)^{(n-3) / 2} d x} .
$$

Пусть $\delta \in(0,1)$ и $[a, b]=[\delta r, r]$. Воспользовавшись, например, методом Лапласа вычисления асимптотики интеграла по большому параметру, из (1) находим, что при $n \rightarrow \infty$

$$
\operatorname{Pr}_{n}[\delta r, r] \sim \frac{1}{\delta \sqrt{2 \pi n}} e^{-\delta^{2} n / 2}
$$

Это уже количественное выражение сделанного выше наблюдения, что подавляющая часть площади многомерной сферы сосредоточена в малой окрестности экваториальной плоскости - в слое $S_{[-\delta r, \delta r]}^{n-1}(r)$ над отрезком $[-\delta r, \delta r]$. Оценка сверху отношения $S_{[-\delta r, \delta r]}^{n-1}(r) / S_{[-r, r]}^{n-1}(r)$, которую можно провести, полагая $r=1$, показывает, что, например,

$$
\operatorname{Pr}_{n}[-\delta r, \delta r]>1-\sqrt{\frac{\pi}{2}} e^{-\delta^{2} n / 2}
$$

Отсюда, в частности, вытекает, что если в $\mathbf{R}^{n}$ взять случайно и независимо пару единичных векторов $v_{1}, v_{2}$, то при $n \gg 1$ с большой вероятностью они окажутся почти ортогональными, т.е. их скалярное произведение $\left\langle v_{1}, v_{2}\right\rangle$ будет близко к нулю. А именно,

$$
\operatorname{Pr}_{n}\left\{\left|\left\langle v_{1}, v_{2}\right\rangle\right|>\delta>0\right\}<\sqrt{\frac{\pi}{2}} e^{-\delta^{2} n / 2} .
$$

Из (2) и (3) видно, что при $n \gg 1$ типичные значения случайной величины $\left|\left\langle v_{1}, v_{2}\right\rangle\right|$ будут порядка $1 / \sqrt{n}$. В случае сферы радиуса $\sqrt{n}$ и векторов $V_{1}, V_{2}$ длины $\sqrt{n}$ стандартное отклонение величины $\left\langle V_{1}, V_{2}\right\rangle$ от ее среднего значения (нуля) будет порядка 1.

Развивая проделанные вычисления, вернемся к соотношению (1). Полагая в нем $r=\sigma \sqrt{n}$ и переходя к пределу при $n \rightarrow \infty$, приходим к классическому нормальному закону распределения вероятностей:

$$
\operatorname{Pr}_{n}[a, b] \rightarrow \frac{1}{\sqrt{2 \pi} \sigma} \int_{a}^{b} e^{-x^{2} /\left(2 \sigma^{2}\right)} d x
$$

Мы получили распределение вероятностей значений координаты случайной точки сферы $S^{n-1}(\sigma \sqrt{n}) \subset \mathbf{R}^{n}$ при $n \gg 1$.

Но отсюда с очевидностью вытекают и закон Гаусса распределения ошибок измерений, и законы Максвелла распределения молекул газа по 
скоростям и энергиям (мы считаем в первом случае, что измерения независимы, имеют нулевое среднее значение и их среднее квадратичное отклонение стабилизируется с ростом количества наблюдений, а во втором случае, что газ однороден и совокупная кинетическая энергия $E_{n}$ молекул порции газа пропорциональна количеству $n$ молекул в этой порции, т.е. $E_{n}=\sigma^{2} n, r_{n}^{2}=E_{n}$, и делается предельный переход при $\left.n \rightarrow \infty\right)$.

Мы заметили также, что при $n \gg 1$ функция $\left\langle v_{1}, v_{2}\right\rangle$ с точки зрения наблюдателя, измеряющего ее значения в случайных местах области определения, практически постоянна. Рассмотрим это явление подробнее.

2. Функции очень многих переменных. Пусть $f$ - дифференцируемая вещественнозначная функция, определенная в некоторой области пространства $\mathbf{R}^{n}$, например, в шаре $B^{n}$. В соотношении $f(x+h)-f(x)=f^{\prime}(x) h+o(h)$, определяющем дифференцируемость функции $f$ в точке $x \in B^{n}$, касательное отображение $f^{\prime}(x)$ линейно относительно вектора смещения $h$. При желании $f^{\prime}(x) h$ можно интерпретировать как скалярное произведение $\langle\operatorname{grad} f(x), h\rangle$. Допустим теперь (как это типично бывает, например, в статистической термодинамике), что $n \gg 1$, т.е. что мы имеем дело с функцией очень многих равноправных переменных. В этом случае, как мы заметили выше, вектор смещения $h$, как правило, будет почти ортогонален фиксированному вектору $\operatorname{grad} f(x)$. Тогда $f^{\prime}(x) h=0$, и для наблюдаемых значений аргументов имеем $f(x+h)=f(x)$. Мы здесь пренебрегли величиной $o(h)$, но можно было бы, обратившись к теореме Лагранжа о конечном приращении, написать $f(x+h)-f(x)=f^{\prime}(\xi) h$ и повторить аргументацию применительно к $\langle\operatorname{grad} f(\xi), h\rangle$.

Эти прикидки поясняют, почему с точки зрения наблюдателя функции очень многих переменных (такие, например, как температура или давление в термодинамике) практически постоянны или, во всяком случае, очень стабильны. В теории вероятностей это явление (применительно к линейным функциям) хорошо известно и именуется законом больших чисел.

Если бы точка $x$ случайным образом дрейфовала по области под действием белого шума, а $\Delta$ был бы случайным вектором смещения из состояния $x$, то с большой вероятностью значение $f^{\prime}(x) \Delta$ было бы почти нулем, если $n \gg 1$, причем эта вероятность экспоненциально стремилась бы к 1 с ростом $n$. Таким образом, дрейф долго проходил бы вдоль уровней функции. Но как это может быть, если он происходит, не зная о существовании функции? Дело в том, что слоение уровнями функции это слоение малой коразмерности (в данном случае коразмерности 1) в пространстве очень большой размерности. Эффект, конечно, будет тем же, если взять не одну, а несколько (но не слишком много) таких функций. 
В частности, это означает, например, что у больших гамильтоновых динамических систем интегралы по существу оказываются функциями гамильтониана в указанном выше смысле.

Мы здесь говорили о функции, предполагая какую-то ее регулярность, невырожденность и, например, непрерывность и ограниченность градиента. Большие величины градиента, его разрывы или разрывы следующей производной, конечно, могут резко менять описанную картину. Возможные критические ситуации, разумеется, требуют более тщательного рассмотрения.

Вернемся теперь еще раз на сферу и конкретизируем сделанные здесь общие наблюдения.

3. Многомерная сфера, закон больших чисел и центральная предельная теорема. Пусть $S^{m}$ - единичная сфера в евклидовом пространстве $\mathbf{R}^{m+1}$ очень большой размерности $m+1$. Пусть на сфере задана достаточно регулярная (например, из некоторого фиксированного класса Липшица) вещественнозначная функция. Берем случайно и независимо друг от друга пару точек на сфере и вычисляем в них значения нашей функции. С большой вероятностью эти значения будут почти одинаковы и близки к некоторому числу $M_{f}$.

Это, пока еще гипотетическое, число $M_{f}$ называют медианньлм значением функиии или медианой функиии. Его также называют средним значением функиии в смысле Леви. Мотивировка терминов вскоре прояснится вместе с точным определением $M_{f}$.

Поясним этот полезный и важный во многих отношениях (например, для статистической физики) факт.

Введем некоторые обозначения и соглашения. Договоримся расстояние между точками сферы $S^{m} \subset \mathbf{R}^{m+1}$ понимать в смысле ее геодезической метрики $\varrho$. Через $A_{\delta}$ обозначим $\delta$-окрестность в $S^{m}$ множества $A \subset S^{m}$. Поднормируем стандартную меру сферы, заменив ее равномерно распределенной вероятностной мерой $\mu$, т.е. $\mu\left(S^{m}\right)=1$.

Справедливо следующее утверждение, доказанное Полем Леви и именуемое обычно изопериметрическим неравенством Леви: Для любьх $0<a<1$ и $\delta>0$ существует $\min \left\{\mu\left(A_{\delta}\right): A \subset S^{m}, \mu(A)=a\right\} u$ он достигается на сферической шапочке $A^{\circ}$ меры а. Здесь $A^{o}=B(r)$, где $B(r)=B\left(x_{o}, r\right)=\left\{x \in S^{m}: \varrho\left(x_{o}, x\right)<r\right\}$ и $\mu(B(r))=a$.

При $a=1 / 2$, т.е. когда $A^{o}$ - полусфера, получаем следствие: $е с л и$ подмножество $A \subset S^{n+1}$ таково, что $\mu(A) \geqslant 1 / 2, \operatorname{mo} \mu\left(A_{\delta}\right) \geqslant 1-$ $\sqrt{\pi / 8} e^{-\delta^{2} n / 2}$

При $n \rightarrow \infty$ здесь $\sqrt{\pi / 8}$ можно заменить на $1 / 2$.

Теперь обозначим через $M_{f}$ такое число, для которого

$$
\mu\left\{x \in S^{m}: f(x) \leqslant M_{f}\right\} \geqslant \frac{1}{2} \quad \text { и } \quad \mu\left\{x \in S^{m}: f(x) \geqslant M_{f}\right\} \geqslant \frac{1}{2} .
$$


Его-то и называют медианой или средним в смысле Леви значением функции $f: S^{m} \rightarrow \mathbf{R}$. (Если $M_{f}$-уровень функции $f$ на сфере имеет нулевую меру, то мера каждого из указанных двух множества будет в точности равна половине $\mu$-площади сферы $S^{m}$.)

Следующее утверждение, смысл которого разъяснен выше, называется леммой Леви: Если $f \in C\left(S^{n+1}\right)$ u $A=\left\{x \in S^{n+1}: f(x)=M_{f}\right\}$, $\operatorname{mo} \mu\left(A_{\delta}\right) \geqslant 1-\sqrt{\pi / 2} e^{-\delta^{2} n / 2}$.

Мы начали все изложение с замечания, что почти вся площадь многомерной сферы сосредоточена в окрестности экватора. Теперь мы имеем общее утверждение той же природы: малая окрестность медианного уровня любой функции, непрерывной на многомерной сфере, содержит почти всю сферу. Именно поэтому с точки зрения наблюдателя, измеряющего значения такой функции, она и представляется практически постоянной. Проделаем некоторые вычисления, которые количественно пояснят сказанное.

Пусть $\omega_{f}(\delta)=\sup \{|f(x)-f(y)|: \varrho(x, y) \leqslant \delta\}-$ модуль непрерьвности функции $f$. Значения функции $f$ на множестве $A_{\delta}$ близки к $M_{f}$. Точнее, если $\omega_{f}(\delta) \leqslant \varepsilon$, то $\left|f(x)-M_{f}\right| \leqslant \varepsilon$ на $A_{\delta}$. Таким образом, лемма Леви показывает, что «хорошие» функции почти постоянны на почти всей области определения - единичной сфере $S^{m}$, когда ее размерность $m$ очень велика. Точнее, из леммы Леви при $m=n+1$ получаем оценку $\operatorname{Pr}\left\{\left|f(x)-M_{f}\right|>\omega_{f}(\delta)\right\}<\sqrt{\pi / 2} e^{-\delta^{2} n / 2}$.

Например, если $f \in \operatorname{Lip}\left(S^{n+1}, \mathbf{R}\right)$ и $L-$ константа Липшица для функции $f$, то $\operatorname{Pr}\left\{\left|f(x)-M_{f}\right|>\delta / L\right\}<\sqrt{\pi / 2} e^{-\delta^{2} n / 2}$ или, в иной записи, $\operatorname{Pr}\left\{\left|f(x)-M_{f}\right|>\varepsilon\right\}<\sqrt{\pi / 2} e^{-(\varepsilon / L)^{2} n / 2}$. При этом стандартное отклонение величины $\left|f(x)-M_{f}\right|$ от нуля, если $n \gg 1$, будет порядка $L / \sqrt{n}$.

В случае, когда функция $f$ определена не на единичной сфере, а на сфере радиуса $r$, величина $r$ войдет в проведенные оценки. Например, $\varepsilon / L$ заменится на $\varepsilon /(r L)$, а стандартное отклонение значений функции от $M_{f}$ естественно возрастет пропорционально $r$.

Если $f$ - гладкая функция, то константой Липшица $L$ для нее, очевидно, может служить максимум модуля ее градиента. Так, для линейной функции $S_{n}=\left(x_{1}+\cdots+x_{n}\right) / n$ имеем $L=L_{n}=1 / \sqrt{n}$.

Возьмем последовательность $f_{n} \in \operatorname{Lip}\left(S^{n-1}\left(r_{n}\right), \mathbf{R}\right)$ липшицевых функций, для которых $L_{n}=O(1 / \sqrt{n})$ при том, что $r_{n}=\sqrt{n}$. Учитывая только что сказанное, получим, что $\operatorname{Pr}\left\{\left|f_{n}(x)-M_{f_{n}}\right|>\varepsilon\right\}<$ $\sqrt{\pi / 2} e^{-\left(\varepsilon /\left(r_{n} L_{n}\right)\right)^{2} n / 2}$, а для стандартного отклонения от нуля величины $\left|f_{n}(x)-M_{f_{n}}\right|$ имеем верхнюю оценку $r_{n} L_{n} / \sqrt{n}=O(1 / \sqrt{n})$ при $n \gg 1$.

В частности, когда $f_{n}=S_{n}$, получаем классический закон больших чисел, относящийся к значениям простейшей линейной функции $S_{n}=$ $\left(x_{1}+\cdots+x_{n}\right) / n$ при $n \gg 1$.

Пусть $f_{n}=x_{1}+\cdots+x_{n}$. Уровни этой функции - гиперплоско- 
сти в $\mathbf{R}^{n}$, ортогональные вектору $(1, \ldots, 1)$. То же можно сказать и о линейной функции $\Sigma_{n}=\left(x_{1}+\cdots+x_{n}\right) / \sqrt{n}$ с той разницей, что при движении из начала координат в направлении $(1, \ldots, 1)$ ее значения совпадают с расстоянием до начала координат. По этой причине на сфере $S^{n-1}\left(r_{n}=\sigma \sqrt{n}\right)$ они распределены так же, как любая из координат. Но предельное (при $n \rightarrow \infty)$ распределение вероятностей координат нам уже известно (формула (4)). Получаем вариант центральной предельной теоремы теории вероятностей. Конечно, все сказанное относится вообще к распределению значений скалярного произведения $\langle e, x\rangle$ вектора $x \in S^{n-1}\left(r_{n}=\sigma \sqrt{n}\right)$ и единичного вектора $e \in \mathbf{R}^{n}$ при $n \rightarrow \infty$.

Выше мы рассматривали в термодинамическом пределе (при $n \rightarrow \infty)$ распределение проекции сферы $S^{n-1}\left(r_{n}=\sigma \sqrt{n}\right)$ на прямую. Но все можно повторить, рассмотрев проекцию сферы на подпространство любой фиксированной конечной размерности. В пределе при $n \rightarrow \infty$ получим то, что в термодинамике соответствует положению Гиббса: малая часть большой, находящейся в равновесии термодинамической системы, распределена по энергии канонически (нормально).

Отметим еще, что рассматривать при $n \rightarrow \infty$ распределение проекции сферы $S^{n-1}\left(r_{n}=\sigma \sqrt{n}\right)$ на прямую при условии, что радиус сферы растет как $\sqrt{n}$, подсказывает и статистическая термодинамика (когда совокупная кинетическая энергия большого числа молекул пропорциональна их количеству), и статистика обработки не очень грубых измерений (когда ограничена их средняя квадратичная погрешность), и геометрия (которая показывает, что шар единичного объема или массы в пространстве $\mathbf{R}^{n}$ асимптотически при $n \gg 1$ имеет радиус $\left.\sqrt{n /(2 \pi e)}\right)$.

Вместе с тем оценку $\operatorname{Pr}\left\{\left|f_{n}(x)-M_{f_{n}}\right|>\varepsilon\right\}<\sqrt{\pi / 2} e^{-\left(\varepsilon /\left(r_{n} L_{n}\right)\right)^{2} n / 2}$, очевидно, можно использовать и при другом сочетании величин $r_{n}, L_{n}$, например, для получения в рассматриваемой ситуации усиленного закона больших чисел.

В нашем специальном случае координаты $\left(x_{1}, \ldots, x_{n}\right)$ точки $x$ сферы $S^{n-1}\left(r_{n}\right)$ распределены одинаково. Если их дисперсии суть $\alpha_{n}^{2} n$, то равенство $x_{1}^{2}+\cdots+x_{n}^{2}=r_{n}^{2}$ означает, что $r_{n}=\alpha_{n} n$. Пусть, например, $\alpha_{n}^{2}=(\ln n \cdot \ln \ln n)^{-1}$. Пользуясь оценкой $\operatorname{Pr}\left\{\left|f_{n}(x)-M_{f_{n}}\right|>\varepsilon\right\}<$ $\sqrt{\pi / 2} e^{-\left(\varepsilon /\left(r_{n} L_{n}\right)\right)^{2} n / 2}$ применительно к функции $S_{n}=\left(x_{1}+\cdots+x_{n}\right) / n$, можно проверить сходимость ряда $\sum_{n=1}^{\infty} \operatorname{Pr}\left\{\left|S_{n}\right|>\varepsilon\right\}$ при любом $\varepsilon>0$, что и гарантирует выполнение равенства $\operatorname{Pr}\left\{S_{n} \rightarrow 0\right\}=1$, соответствующего усиленному закону больших чисел.

Напомним, что общее и близкое к необходимому достаточное условие Колмогорова для выполнимости усиленного закона больших чисел $\operatorname{Pr}\left\{S_{n}=\left(\xi_{1}+\cdots+\xi_{n}\right) / n \rightarrow 0\right\}=1$ для последовательности независимых не обязательно одинаково распределенных центрированных случайных величин $\xi_{1}, \ldots, \xi_{n}, \ldots$ состоит в сходимости ряда $\sum_{n=1}^{\infty} n^{-2} \mathbf{D} \xi_{n}$, где $\mathbf{D} \xi_{n}$ - дисперсия случайной величины $\xi_{n}$. 
Если, исходя из рассмотренной выше последовательности случайных векторов $\left(x_{1}, \ldots, x_{n}\right)$ растущей размерности, взять от каждого только одну координату, то можно построить диагональную последовательность $x_{1}^{1}, \ldots, x_{n}^{n}, \ldots$ случайных величин, для которой будет выполнен усиленный закон больших чисел, хотя достаточное условие приведенной теоремы будет нарушено (правда, нарушение будет уже на логарифмическом, а не на степенном уровне асимптотики).

4. Замечание об эргодичности. Доказательство эргодичности конкретной конечномерной динамической системы, как правило, дело тонкое. Но в приложениях, например для термодинамики, обычно интерес представляет то, что получается при так называемом термодинамическом предельном переходе, когда размерность неограниченно растет (растет число частиц и фазовый объем системы при сохранении удельной плотности, например, газа).

Рассмотрим однородный идеальный газ в какой-то емкости и какуюлибо функцию (типа давления или температуры), определяемую состоянием движения молекул газа. Пусть $x_{1}, \ldots, x_{n}-$ скорости молекул. Естественно считать, что совокупная кинетическая энергия $E_{n}$ молекул пропорциональна их количеству $n$, т.е.

$$
x_{1}^{2}+\cdots+x_{n}^{2}=\sigma^{2} n .
$$

Если $x_{1}, \ldots, x_{n}$ - трехмерные векторы, то соотношение (5) определяет сферу радиуса $\sigma \sqrt{n}$ в пространстве $\mathbf{R}^{3 n}$. Наша функция $f$ оказывается функцией точки $x$ на этой сфере.

Если $n \gg 1$, что естественно предполагается, а функция $f$ регулярна, то (после изложенного выше) понятно, что она очень стабильна: будут наблюдаться постоянные значения $f$, несмотря на разнообразие комбинаций значений $x_{1}, \ldots, x_{n}$, связанных соотношением (5).

Если бы мы имели динамическую систему $x=x(t)$, эволюционирующую по этой сфере (для которой она была бы уровнем энергии), то для большинства траекторий невырожденной динамической системы (общего положения) любая регулярная функция на сфере представлялась бы почти интегралом движения, ибо подавляющая часть траектории оказывается в окрестности медианного значения $M_{f}$ функции $f$. Разумеется, среднее по траектории общего положения и среднее по пространству (по сфере) тут совпадают и равны $M_{f}$, когда $n \gg 1$.

Эргодическая гипотеза статистической механики, как известно, идет от Больцмана и связана с двумя способами наблюдения (измерения) величин: брать среднее значение величины по всей совокупности однотипных объектов или следить за случайно выбранным типичным объектом и брать среднее по времени от значения этой величины вдоль траектории этого объекта. 
Довольно скоро было понято, что для выводов теории столь сильное предположение, как эргодичность динамической системы, совсем не обязательно. Важна только концентрация меры, связанная с тем, что типичные системы статистической термодинамики содержат очень много частиц (очень многомерны). Лоренц это отмечает совершенно явно: «Максимумы же вероятности, входящие в теории, которыми мы занимаемся, чрезвычайно остры» (см. лекции Лоренца «Статистические теории в термодинамике» [8]). Это и значит, что плотности распределения вероятности состояний системы и значений наблюдаемых величин очень концентрированы, поэтому мы получаем стабильные значения измеряемых функций состояния системы, хотя, в отличие от феноменологической термодинамики, статистическая механика не исключает больших уклонений, указывая одновременно малость их вероятности.

5. Гауссовские меры и их концентрация. а. В связи с обсуждением наблюдаемой стабилизации (постоянства) значений регулярной функции на многомерной сфере мы сказали об изопериметрическом неравенстве на сфере. Такой же вопрос о минимизации меры $\delta$-раздутия множества важен и по тем же причинам интересен также по отношению к другим пространствам, которые служат естественными областями определения нужных функций.

Например, для гауссовских вероятностных мер, определяемых нормальным законом распределения вероятностей $N(0,1)$ в стандартном евклидовом пространстве $\mathbf{R}^{n}$, ответ на этот вопрос тоже известен (см., например, [6], [7]). В этом случае экстремальной областью (с исходно фиксированным значением гауссовской меры и $\delta$-раздутием, понимаемым в евклидовой метрике) оказывается полупространство.

В частности, если взять полупространство гауссовской меры $1 / 2$ и непосредственно вычислить значение гауссовской меры дополнения к его евклидову $\delta$-раздутию, то, учитывая упомянутое изопериметрическое неравенство, можно заключить, что для любого множества $A$, имеющего в пространстве $\mathbf{R}^{n}$ гауссовскую меру $1 / 2$, меру его $\delta$-раздутия можно оценить снизу следующим образом: $\mu\left(A_{\delta}\right) \geqslant 1-I_{\delta}$, где $I_{\delta}$ - интеграл от плотности $(2 \pi)^{-n / 2} \exp \left(-|x|^{2} / 2\right)$ гауссовской меры по полупространству, удаленному от начала координат на евклидово расстояние $\delta$.

Оценка интеграла $I_{\delta}$ сверху позволяет, например, утверждать, что $\mu\left(A_{\delta}\right) \geqslant 1-2 \exp \left(-\delta^{2} / 2\right)$.

b. Это грубая оценка, но и она показывает быстрый рост $\mu\left(A_{\delta}\right)$ при увеличении $\delta$ каково бы ни было исходное множество $A$ меры $1 / 2$.

Очень интересно (а учитывая возможные переходы в бесконечномерные пространства, даже весьма полезно) заметить, что последняя оценка не зависит от размерности пространства. Может показаться, что отсутствие здесь размерности пространства - большая потеря и что эта оценка слаба в контексте обсуждаемой концентрации меры и 
стабилизации значений функций многих переменных. На самом деле эта оценка фактически содержит в себе даже результат о концентрации меры на единичной сфере большой размерности, обсуждавшийся выше.

Достаточно проверить, что основная часть гауссовской вероятностной меры евклидова пространства $\mathbf{R}^{n}$ при $n \gg 1$ сосредоточена в единичной окрестности евклидовой сферы радиуса $\sqrt{n}$. Значит, в пересечении этой окрестности с полупространством, заметно удаленным от начала координат, доля этой меры экспоненциально мала. Таким образом, основная часть меры оказывается в той части окрестности сферы радиуса $\sqrt{n}$, которая попадает в слой между двумя близкими параллельными гиперплоскостями, симметричными относительно начала координат. Если, сделав это наблюдение, теперь гомотетией перейти от сферы радиуса $\sqrt{n}$ к единичной сфере, то получится принцип концентрации меры на единичной сфере, который мы обсуждали выше. В нем размерность пространства присутствует явно. Она присутствовала и в гауссовском случае, но была спрятана в размере $\sqrt{n}$ сферы, в окрестности которой сосредоточена основная доля меры всего пространства.

6. Еще немного о многомерном кубе. В евклидовом пространстве $\mathbf{R}^{n}$ рассмотрим $n$-мерный единичный промежуток («куб») $I^{n}:=$ $\left\{x=\left(x^{1}, \ldots, x^{n}\right) \in \mathbf{R}^{n}:\left|x^{i}\right| \leqslant 1 / 2, i=1,2, \ldots, n\right\}$.

Его объем равен 1 , хотя диаметр равен $\sqrt{n}$. (Напомним, кстати, что объем $n$-мерного шара радиуса $r$ в евклидовом пространстве $\mathbf{R}^{n}$ выражается формулой

$$
V_{n}(r)=\frac{\pi^{n / 2}}{(n / 2) \Gamma(n / 2)} r^{n}
$$

из которой следует, что главный член асимптотики величины $r_{n}$ радиуса $n$-мерного шара единичного объема при $n \rightarrow+\infty$ имеет вид $\sqrt{n /(2 \pi e)}$, т.е. это величина порядка $\sqrt{n}$.) Будем считать стандартную меру вероятностной равномерно распределенной мерой на кубе $I^{n}$.

Пусть $a=\left(a^{1}, \ldots, a^{n}\right)$ - единичный вектор, а $x=\left(x^{1}, \ldots, x^{n}\right)$ произвольная точка куба $I^{n}$.

Справедливо следующее неравенство (оценка вероятности типа неравенства Бернштейна): $\operatorname{Pr}_{n}\left\{\left|\sum_{i=1}^{n} a^{i} x^{i}\right| \geqslant t\right\} \leqslant 2 \exp \left(-6 t^{2}\right)$.

Интерпретируя сумму $\sum_{i=1}^{n} a^{i} x^{i}$ как скалярное произведение $\langle a, x\rangle$, понимаем, что оно может быть большим (порядка $\sqrt{n}$ ), если вектор $a$ направить не вдоль какого-то одного ребра куба, а вдоль главной диагонали, смешивая все координатные направления в равной степени. Взяв $a=(1 / \sqrt{n}, \ldots, 1 / \sqrt{n})$, на основании указанной оценки заключаем, что объем $n$-мерного куба $I^{n}$ с ростом $n$ концентрируется в относительно малой окрестности гиперплоскости, проходящей через начало координат ортогонально вектору $(1 / \sqrt{n}, \ldots, 1 / \sqrt{n})$. 
В отличие от случая шара или сферы, на кубе регулярная функция может терять свойство концентрации значений, понимаемое в прежнем смысле, когда вероятность отклонения значения функции от медианного значения убывала экспоненциально; взять хотя бы функцию, значение которой в точке совпадает со значением первой координаты этой точки. Проекция шара на любую прямую устроена одинаково, а в случае куба это не так.

При исследовании концентрации значений липшицевых функций на кубе полезно, однако, иметь в виду следующее обстоятельство: какие бы два множества в кубе $I^{n}$ ни взять, если их объемы больше некоторой величины $\varepsilon$, то расстояние между ними не превосходит величины $d(\varepsilon)$, зависящей только от $\varepsilon$, но не от размерности куба. Нетривиальность утверждения в том, что при этом диаметр куба $\sqrt{n}$ может быть сколь угодно большим. То же, конечно, относится и к шару единичного объема.

7. Кодирование сигнала в канале с шумом. Укажем еще одну область, где функции очень большого числа переменных тоже появляются естественным образом и где принцип концентрации меры проявляется и используется тоже по существу.

Мы уже привыкли к цифровому (дискретному) кодированию и передаче сигнала (музыки, изображения, сообщения - информации) по каналу связи. В таком виде сообщение можно себе мыслить как вектор $x=\left(x^{1}, \ldots, x^{n}\right)$ в пространстве $\mathbf{R}^{n}$ очень большой размерности. На передачу такого сообщения затрачивается энергия $E$, пропорциональная $\|x\|^{2}=\left|x^{1}\right|^{2}+\cdots+\left|x^{n}\right|^{2}$ (подобно рассмотренной выше суммарной кинетической энергии молекул газа). Если $T$ - продолжительность передачи сообщения $x$, то $P=E / T$ - средняя мощность, затрачиваемая на передачу одного символа (одной координаты вектора $x$ ). Если $\Delta-$ среднее время, затрачиваемая на передачу одной координаты вектора $x$, то $T=n \Delta$ и $E=n P \Delta$.

Передающее и принимающее устройства согласованы так, что передатчик преобразует (кодирует) подлежащее передаче исходное сообщение в форму вектора $x$, отправляет его по каналу связи, а приемник, зная код, расшифровывает $x$, преобразуя его в форму исходного сообщения.

Если нам надо передать $M$ сообщений $A_{1}, \ldots, A_{M}$ длины $n$, то достаточно в шаре радиуса $E=n P \Delta$ фиксировать $M$ точек $a_{1}, \ldots, a_{M}$, согласовав этот выбор с приемным концом канала связи. Если в канале связи нет помех, то, получив вектор $а$ из согласованного набора, приемник безошибочно декодирует его в соответствующее сообщение $A$.

Если же в канале связи есть помехи (что обычно и случается), то помеха, случайный вектор $\xi=\left(\xi^{1}, \ldots, \xi^{n}\right)$, сместит передаваемый вектор $a$ и на приемник поступит вектор $a+\xi$, который надо будет правильно расшифровать. 
Если точки $a_{1}, \ldots, a_{M}$ были выбраны так, что шары радиуса $\|\xi\|$ c центрами в этих точках не пересекаются, то однозначная расшифровка еще возможна. Но если соблюдать это требование, то уже нельзя брать сколько угодно точек $a_{1}, \ldots, a_{M}$ и возникает проблема плотной упаковки шаров. Это сложная задача, решения которой в рассматриваемой ситуации, как показал К.Шеннон, можно избежать, учитывая, что размерность $n$ пространства $\mathbf{R}^{n}$ здесь огромна.

Позволим себе иногда ошибаться при расшифровке принятого сообщения. Потребуем, однако, чтобы вероятность ошибки была сколь угодно мала (меньше любого фиксированного положительного числа). Шеннон показал, что даже при наличии в канале связи случайных помех (белый шум) любой ограниченной мощности, выбирая достаточно длинный код (т.е. при больших значениях $n$ ), можно добиться скорости передачи сколь угодно близкой к скорости передачи информации по каналу без шума, при этом со сколь угодно малой вероятностью ошибки.

Геометрическая идея теоремы Шеннона непосредственно связана с обсуждавшимися выше особенностями распределения меры (объема) областей в пространстве большой размерности. Поясним это.

Предположим, что два одинаковых шара в пространстве $\mathbf{R}^{n}$ пересекаются. Если принятый сигнал окажется в этом пересечении, то возможна ошибка в расшифровке переданного сообщения. Но если вероятность попадания в какую-то область считать пропорциональной относительному объему области, то естественно сравнить объем пересечения шаров с объемом шара. Проведем нужную оценку. Если центры двух шаров радиуса 1 находятся на взаимном расстоянии $\varepsilon(0<\varepsilon<2)$, то пересечение этих шаров содержится в шаре радиуса $\sqrt{1-(\varepsilon / 2)^{2}}$ с центром в середине отрезка, соединяющего центры исходных шаров. Значит, отношение объема пересечения двух исходных шаров к собственному объему каждого из них не превосходит $\left(1-(\varepsilon / 2)^{2}\right)^{n / 2}$. Теперь ясно, что при любом фиксированном значении $\varepsilon$ эта величина может быть сделана сколь угодно малой за счет выбора достаточно большого значения $n$.

\section{8. Одна конкретная формулировка принципа концентра-}

ции. «Почти весь объем многомерного тела находится в малой окрестности границы тела». Дадим и докажем теперь возможную конкретную формулировку этого эвристического принципа концентрации меры, согласно которому почти весь объем многомерного тела сосредоточен в малой окрестности его границы. Мы уже показали выше, что это общее обстоятельство имеет разнообразные, хотя не всегда идентифицируемые специалистами, частные проявления (в статистической физике, теории вероятностей, теории динамических систем, геометрии, анализе). Например, оно проявляется в том, что функции очень большого числа переменных имеют тенденцию быть почти постоянными с точки зрения измеряющего их значения наблюдателя. 
Теорема. Для любых положительньх чисел $\varepsilon$ и $\delta$ найдется такое иисло $N$, ито при любом $n>N$ и для любой области $D$, лежащей в единичном шаре пространства $\mathbf{R}^{n}$, справедливо следующее: объем части области $D$, содержащейся в $\delta$-окрестности граниџь области, не меньше чем $(1-\varepsilon)|D|$, где $|D|$ - объем области.

Д о к а з а т е л ь с т в о. Напомним сначала изопериметрическое неравенство Леви [2], [3].

На $n$-мерной сфере рассматриваются области (множества) $D$ фиксированной площади ( $n$-меры) $|D|=c$. Среди них ищется такая, $\delta$ раздутие которой (в смысле геодезической метрики на сфере) имеет минимальную площадь. Изопериметрическое неравенство Леви состоит в том, что такой областью является сферическая шапочка заданной площади $c$.

Поскольку радиус сферы здесь роли не играет, изопериметрическое неравенство Леви справедливо и для областей пространства $\mathbf{R}^{n}$. Впрочем, в этом случае оно получается интегрированием классического изопериметрического неравенства в $\mathbf{R}^{n}$.

Рассмотрим теперь область $D$, лежащую в единичном шаре $\mathbf{B}^{n}$ пространства $\mathbf{R}^{n}$. Пусть $\partial D$ - граница области $D$, а $\Delta=D \cap(\partial D)_{\delta}$ часть области $D$, содержащаяся в $\delta$-окрестности границы области.

Рассмотрим ту часть $d=D \backslash \Delta$ области $D$, которая не попала в $\Delta$, и заметим, что ее $\delta$-раздутие $d_{\delta}$ содержится в $D$.

В силу изопериметрического неравенства Леви величина $\left|d_{\delta} \backslash d\right|$ не меньше, чем величина $\left|b_{\delta} \backslash b\right|$ приращения объема шара $b \subset \mathbf{R}^{n}$, возникающая при $\delta$-раздутии шара $b$, если $|b|=|d|$. Таким образом, $\left|d_{\delta}\right| \geqslant\left|b_{\delta}\right|$ и, тем более, $|D| \geqslant\left|b_{\delta}\right|$. Значит, $(|D|-|d|) /|D| \geqslant\left(\left|b_{\delta}\right|-|b|\right) /\left|b_{\delta}\right|$, или $|\Delta| /|D| \geqslant\left(\left|b_{\delta}\right|-|b|\right) /\left|b_{\delta}\right|$.

Но для шара $b_{\delta} \subset \mathbf{R}^{n}$, с одной стороны, $b=b_{\delta} \backslash\left(\partial b_{\delta}\right)_{\delta}$, а с другой стороны, нам известно, что если радиус шара ограничен, например, единицей, а размерность пространства достаточно большая, то для такого шара утверждение теоремы верно.

Значит, оно верно и для любой области, лежащей в таком шаре.

9. Замечания о первоисточниках и библиографии. Принцип концентрации, его зарождение, развитие, математическое оформление, геометрическое содержание, связи с теорией вероятностей, теорией функций, выпуклым анализом можно проследить по источникам [1]-[7] приводимого ниже списка литературы. Разумеется, это очень ограниченный и условный выбор. Однако он, по-видимому, включался бы почти в каждый список, составленный на данный предмет. Отметим, что в указанных источниках, как правило, имеется дальнейшая библиография, ориентированная в своем направлении, которое может быть особенно интересно читателю. 
Физическая сторона дела, связанная со статистической термодинамикой, линейным и нелинейным законами больших чисел, каноническим распределением, представлена, например, в источниках [8]-[20].

Применения в теории передачи информации по каналу связи при наличии шума (помех) представлены, например, в источниках [21], [22].

В большинстве случаев читатель не найдет там даже упоминания о каком-то общем принципе концентрации, но, будучи подготовлен предыдущим обсуждением, сам увидит его проявление, подобно тому, как, например, физик видит закон сохранения энергии даже в его частных проявлениях.

10. Гауссов нормальный закон распределения и одно замечание Колмогорова. В качестве дополнения к изложенному и в связи с изложенным мы обсудим одно общее наблюдение А. Н. Колмогорова о характере распределения значений произведения очень большого числа функций в окрестности максимума произведения, дадим возможную конкретную формулировку этого наблюдения и докажем его в этой форме.

а. Наблюдение А. Н. Колмогорова. Заинтересовавшись некоторыми математическими аспектами термодинамики, в частности, связью классической термодинамики с контактной геометрией, а статистической термодинамики с многомерной геометрией, я составил себе определенную геометрическую интерпретацию таких фактов теории вероятностей, как закон больших чисел или центральная предельная теорема. Эти законы лежат в основе статистических методов термодинамики, где наблюдаемыми являются статистические средние термодинамических функций. В пространствах очень большой размерности, как было показано выше, имеет место явление концентрации меры: почти весь объем многомерной области сосредоточен в малой окрестности границы или, например, почти вся площадь сферы очень большой размерности сосредоточена в малой окрестности экватора. Это обстоятельство, как мы видели, приводит к стабилизации значений функций очень многих переменных с точки зрения наблюдателя и, в частности, к тому, что все средние (математическое ожидание, медианное значение, ...) наблюдаемой регулярной функции оказываются практически совпадающими. Оно же (явление концентрации меры) часто может успешно заменить гипотезу эргодичности рассматриваемых термодинамических систем.

Не будучи специалистом в теории вероятностей, я обсуждал некоторые вопросы с профессионалами. В одной из таких бесед Альберт Николаевич Ширяев указал мне на письмо А. Н. Колмогорова [23, с. 431]. Я привожу математическую часть этого письма, которую мы потом и обсудим, а также даю ссылку на статью [24], отражающую первую фразу цитаты.

«Хочу заняться восстановлением уважения к методу медианы. Кроме того, из этих исследований извлек некоторые идеи чрезвычайной 
общности, еще не вполне оформленные. Именно, если

$$
\varphi(x)=f_{1}(x) f_{2}(x) \cdots f_{n}(x)
$$

- произведение чрезвычайно большого числа функций и в $x_{0}$ имеет максимум $A=\varphi\left(x_{0}\right)$, то вблизи $x_{0}$

$$
\varphi(x)=A e^{-k\left(x-x_{0}\right)^{2}}
$$

(т.е. Гауссов закон распределения!). Это еще новая причина появления Гауссовой кривой, видимо, совсем отличная от сложения большого числа малых величин! Именно поэтому (при любом основном законе распределения ошибок) ошибка результата измерений (будь то по методу средних арифметических, методу медианы или любому другому) всегда следует закону Гаусса».

\section{b. Интерпретация, формулировка и доказательство утвер- ждения.}

- Сначала тривиальное замечание о представлении регулярной вещественнозначной функции в окрестности точки положительного максимума.

Пусть $F(x)=F(0)+(1 / 2) F^{\prime \prime}(0) x^{2}+o\left(x^{2}\right)$, где $F(0)>0$ и $F^{\prime \prime}(0)<0$.

Тогда в окрестности точки $x=0$

$$
F(x)=\exp \left(\log \left(F(0)+\frac{1}{2} F^{\prime \prime}(0) x^{2}+o\left(x^{2}\right)\right)\right) \simeq F(0) \exp \left(-\frac{\left|F^{\prime \prime}(0)\right|}{2 F(0)} x^{2}\right) .
$$

- Простейшая вероятностная формулировка и пробная проверка исходного утверждения.

Пусть $F_{n}(x)=f^{n}(x)$, где $f(x)=1+(1 / 2) f^{\prime \prime}(0) x^{2}+o\left(x^{2}\right)$, причем $f^{\prime \prime}(0)<0$ и $n \gg 1$. Тогда

$$
\begin{aligned}
F_{n}(x) & =\exp \left(n \log \left(f(0)+\frac{1}{2} f^{\prime \prime}(0) x^{2}+o\left(x^{2}\right)\right)\right) \simeq f(0)^{n} \exp \left(n \frac{f^{\prime \prime}(0)}{2 f(0)} x^{2}\right) \\
& =A e^{-k x^{2}}
\end{aligned}
$$

где

$$
A=f(0)^{n}=1 \quad \text { и } \quad k=-n \frac{f^{\prime \prime}(0)}{2 f(0)}=n\left|\frac{f^{\prime \prime}(0)}{2 f(0)}\right|=n\left|\frac{f^{\prime \prime}(0)}{2}\right| .
$$

- Общий случай.

Пусть

$$
F(x)=f_{1}(x) \cdots f_{n}(x),
$$

где $n \gg 1$, а $f_{k}(x)=f(x)+\varepsilon \varphi_{k}(x), k=1, \ldots, n$, причем величина $\varepsilon$ мала относительно $f(x)$, а функции $\varphi_{k}(x)$ ограничены в совокупности.

Случайность появления значений набора функций $\varphi_{k}(x), k=$ $1, \ldots, n$, пусть состоит в том, что $\sum_{k=1}^{n} \varepsilon \varphi_{k}(x)=n \varepsilon(x)$, где $\varepsilon(x) \leqslant 0$ 
при $n \gg 1$ и $\varepsilon(0)=0$ - максимальное значение функции $\varepsilon(x)$. Предполагаем также, что $f(0)>0$ и $f(0)$ - максимальное значение регулярной функции $f$ в окрестности $x=0$. Тогда

$$
\begin{aligned}
F(x) & =f_{1}(x) \cdots f_{n}(x)=\exp \left(\sum_{k=1}^{n} \log \left(f(x)+\varepsilon \varphi_{k}(x)\right)\right) \\
& =\exp \left(\sum_{k=1}^{n} \log \left(f(0)+\frac{1}{2} f^{\prime \prime}(0) x^{2}+o\left(x^{2}\right)+\varepsilon \varphi_{k}(x)\right)\right) \\
& \simeq f(0)^{n} \exp \left(\sum_{k=1}^{n} \frac{1}{f(0)}\left(\frac{1}{2} f^{\prime \prime}(0) x^{2}+\varepsilon \varphi_{k}(x)\right)\right) \\
& =f(0)^{n} \exp \left(\frac{1}{f(0)}\left(n \frac{1}{2} f^{\prime \prime}(0) x^{2}+n \varepsilon(x)\right)\right) \\
& \simeq f(0)^{n} \exp \left(\frac{1}{f(0)}\left(n \frac{1}{2} f^{\prime \prime}(0) x^{2}+n \frac{1}{2} \varepsilon^{\prime \prime}(0) x^{2}\right)\right) \\
& =f(0)^{n} \exp \left(\frac{n}{2 f(0)}\left(f^{\prime \prime}(0)+\varepsilon^{\prime \prime}(0)\right) x^{2}\right)=A e^{-k x^{2}},
\end{aligned}
$$

где

$$
A=f(0)^{n} \quad \text { и } \quad k=-\frac{n}{2 f(0)}\left(f^{\prime \prime}(0)+\varepsilon^{\prime \prime}(0)\right) .
$$

Заметим, что, меняя масштаб (точнее, полагая $t:=\sqrt{n} x)$, при $n \rightarrow$ $\infty$ мы переходим от окрестности нуля по $x$ на всю ось переменной $t$.

Наблюдение А.Н. Колмогорова, которое мы здесь обсуждали, возможно, допускает иное толкование. Более того, даже приведенная его интерпретация, вероятно, допускает содержательные расширения.

Я признателен Альберту Николаевичу Ширяеву как за исходное указание на рассмотренное здесь наблюдение А.Н. Колмогорова, так и за полезные доброжелательные обсуждения с не очень к ним подготовленным собеседником.

\section{СПИСОК ЛИТЕРАТУРЫ}

Математические первочсточники и их развитие.

1. Poincaré H. Calcul des probabilités. Paris: Gauthier-Villars, 1912, 340 p.; pyc. пер.: Пуанкаре А. Теории вероятностей. М.-Ижевск: РХД, 1999, 276 с.

2. Lévy P. Problèmes concrets d'analyse fonctionnelle. Paris: Gauthier-Villars, 1951, $484 \mathrm{p}$.

3. Milman V., Schechtman G. Asymptotic Theory of Finite Dimensional Normed Spaces. (With an Appendix by M. Gromov.) Berlin: Springer-Verlag, 1986, 156 p. (Lecture Notes in Math. v. 1200.) (Appendix I: M. Gromov, Isoperimetric inequality in Riemannian manifolds, p. 114-129.)

4. Мильман В. Д. Явления, возникающие в высоких размерностях. - Успехи матем. наук, 2004, т. 59, № 1, с. 157-168. 
5. Milman E. On the role of convexity in isoperimetry, spectral gap and concentration. Invent. Math., 2009, v. 177, № 1, p. 1-43.

6. Богачёв В. И. Гауссовские меры. М.: Наука, 1997, 352 с.

7. Ball K. An elementary introduction to modern convex geometry. - Flavors of Geometry. Ed. by S. Lévy. Cambridge: Cambridge Univ. Press, 1997, p. 1-58. (Math. Sci. Res. Inst. Publ., v. 31.)

Физические источники и их математическое развитие.

8. Лорени Г.А. Статистические теории в термодинамике. М.-Ижевск: РХД, 2001, $192 \mathrm{c}$.

9. Шрёдингер Э. Лекции по физике. М.-Ижевск: РХД, 2001, 156 с.

10. Хинчин А.Я. Симметрические функции на многомерных поверхностях. - Памяти Александра Александровича Андронова. М.: Изд-во АН СССР, 1955, с. 541576.

11. Опойцев В. И. Нелинейный закон больших чисел. - Автомат. и телемех., 1994, № 4 , c. $65-75$.

12. Манин Ю.И. Математика и физика. М.: Знание, 1979, 63 с. (Новое в жизни, науке, технике. Сер. Матем., кибернет., в. 12.) Имеется также в кн.: Манин Ю. И. Математика как метафора. М.: МЦНМО, 2008, 400 с.

13. Минлос Р. А. Введение в математическую статистическую физику. М.: МЦНМО, 2002, $111 \mathrm{c}$.

14. Березин Ф. А. Лекции по статистической физике. М.-Ижевск: Институт компьютерных исследований, 2002, 192 с.

15. Синай Я.Г. Теория фазовых переходов. Строгие результаты. М.-Ижевск: РХД, $2001,237 \mathrm{c}$.

16. Рюэль Д. Случайность и хаос. М.-Ижевск: РХД, 2001, 191 с.

17. Кач M. Вероятность и смежные вопросы в физике. М.: УРСС, 2003, 273 с.

18. Козлов В. В. Тепловое равновесие по Гиббсу и Пуанкаре. М.-Ижевск: РХД, 2002, $319 \mathrm{c}$.

19. Kurchan J., Laloux L. Phase space geometry and slow dynamics. - J. Phys. A: Math. Gen., 1996, v. 29, № 9, p. 1929-1948.

20. Zorich V. Mathematical Analysis of Problems in the Natural Sciences. Berlin: Springer, 2011, 135 р. Дополненный перевод на английский язык книги: Зopuч B. A. Математический анализ задач естествознания. М.: МЦНМО, 2008, $135 \mathrm{c}$.

Первоисточники к разделу, связанному с теорией информачии.

21. Шеннон K. Работы по теории информации и кибернетике. М.: ИЛ, 1963, 829 с. Книга включает статью: Шеннон $K$. Математическая теория связи. (Shannon C. E. A mathematical theory of communication. - Bell System Tech. J., 1948, v. 27, p. 379-423, 623-656.)

22. Теория информации и ее приложения. Сб. переводов. Ред. А. А. Харкевич. М.: Физматгиз, 1959, 328 с. Сборник переводов включает статью: Шеннон К. Связь при наличии шума. (Shannon C. E. Communication in the presence of noise. - Proc. IRE, 1949, v. 37, № 1, p. 10-21.)

Литература $к$ дополнению.

23. Колмогоров A.H. Юбилейное издание в трех книгах (к 100-летию со дня рождения акад. А.Н. Колмогорова). Кн. 2: Этих строк бегущих письма. М.: Физматлит, 2003, $671 \mathrm{c.}$

24. Колмогоров А.Н. Метод медианы в теории ошибок. - Матем. сб., 1931, т. 38, № 3-4, с. 47-50. Опубл. также в кн.: Колмогоров А.Н. Теория вероятностей и математическая статистика. М.: Наука, 1986, с. 111-114.

Поступила в редакцию 20.V.2014 